\title{
Elastic strains influence during GaSb/InAs heteroepitaxy from liquid phase
}

\author{
S.V. Shutov', Ye.A. Baganov ${ }^{2}$ \\ ${ }^{I} V$. Lashkaryov Institute of Semiconductor Physics, NAS of Ukraine, \\ 41, prospect Nauky, 03028 Kyiv, Ukraine \\ E-mail: shutov_sv@mail.ru,phone/fax: +380 (552) 515457 \\ ${ }^{2}$ Kherson National Technical University, 24, Berislavskoye shosse, 73008 Kherson, Ukraine \\ E-mail: ewgb@newmail.ru,phone: +380(552) 326922
}

\begin{abstract}
Influence of strains that appear in $\mathrm{GaSb} / \mathrm{InAs}$ heterosystem on heteroepitaxial layer planarity is considered. It is shown that minimal supercooling of solution-melt at the saturation temperature of gallium antimonide in gallium melt $450{ }^{\circ} \mathrm{C}$ is $7.8{ }^{\circ} \mathrm{C}$ for [111] and $5.8^{\circ} \mathrm{C}$ for [100] growth directions. Calculated are the minimal growth rate $22 \mathrm{~nm} / \mathrm{s}$ that is necessary for prevention of distortion appearance of epitaxial layer surface caused by elastic strains and the critical thicknesses of misfit dislocation formation -50 and $54 \mathrm{~nm}$ for the [100] and [111] growth directions, respectively. It is shown experimentally that the lack of minimal supercooling leads to the island growth mode.
\end{abstract}

Keywords: liquid phase epitaxy, strain, growth rate, GaSb, InAs.

Manuscript received 30.11.05; accepted for publication 15.12.05.

\section{Introduction}

Recently the properties of type-II broken gap heterojunction based on InAs and GaSb are widely used for different optoelectronic devices manufacturing [1]. At present, the basic methods used for obtaining $\mathrm{GaSb} / \mathrm{InAs}$ are molecular beam epitaxy (MBE) and metal organic chemical vapour deposition (MOCVD) [2, 3].

Most close to equilibrium growth conditions are provided with methods of liquid phase epitaxy (LPE) [4]. At the same time, there are several specific problems in the course of obtaining GaSb/InAs with the LPE methods. One of the problems is related with interaction between the substrate and liquid phase with non-equilibrium composition. It leads to substrate erosion, non-planarity and uncontrolled composition of the epitaxial layer [5, 6]. Such problem can be solved by LPE from an initially supercooled liquid phase [6]. The second type of problems is connected with availability of elastic strains in the $\mathrm{GaSb} / \mathrm{InAs}$ heterostructure owing to lattice constants mismatch $\left(0.74 \%\right.$ at the epitaxy temperature $\left.450{ }^{\circ} \mathrm{C}\right)$.

The availability of elastic strains in the epitaxial layer, when it grows isomorphously until appearance of misfit dislocations, imposes a requirement on minimal solution-melt supercooling $\Delta T_{\min }$ due to increasing the solid phase chemical potential. When $\Delta T$ values of supercooling are smaller than $\Delta T_{\min }$ the epitaxial layer grows in accordance with the Stranski-Krastanov mechanism [7]. As a result, layer planarity can be broken, to avoid the epitaxial layer non-planarity the supercooling that is sufficient for suppression of Stranski-Krastanov growth mechanism must take place until the layer thickness reaches a critical value of misfit dislocation formation. The elastic strains are driving forces for surface distortion of epitaxial layer, too [8, 9]. Process of suppression of such distortion development can be provided due to the sufficient epitaxial layer growth rates.

In this connection, the aim of this work is calculation of minimal supercooling $\Delta T_{\min }$ and necessary growth rate for optimization of GaSb growth on InAs substrate from liquid phase as well as providing continuous character of the epitaxial layer and its planarity.

\section{Calculation model and results}

$\mathrm{GaSb} / \mathrm{InAs}$ heteroepitaxial layer critical thickness calculated using the Matthews and Blakeslee method [10] is significantly less than the experimental ones [1113]. That is why, to determine the epitaxial layer critical thickness, used was a two-dimensional simulation of elastic strains and deformation energy distribution in the strained structure with an available network of misfit dislocations. 
Simulation by the finite-element method was based on the Van-der-Merwe heteroepitaxial layer growth model [14]. But use of any adequate forces model on the substrate-epitaxial layer interface (such as the PeierlsNabarro potential) leads to nonlinear or transcendent combined equations in the course of simulation. To solve this system the intermediate layer was introduced. The initial structure for simulation (Fig. 1) consisted of the unstrained epitaxial layer, unstrained substrate and strained intermediate layer, one side of which was fitted to the substrate lattice parameter and other one - to the unstrained epitaxial layer lattice parameter. Physical properties of this intermediate layer were defined by averaging those of the substrate and epitaxial layer. From [14], an atom located at the central line of symmetry can be considered as horizontally undisplaced. The misfit dislocation appears when the displacement between the substrate and layer atoms reaches a half of the lattice parameter.

As a critical epitaxial layer thickness, we assumed such thickness value that provides the equality of energies between two-dimensional strained epitaxial layers without dislocations and that with misfit dislocations. The energy of the elastically strained twodimensional epitaxial layer is determined as follows:

$F=\frac{1}{2} \frac{E U_{x x}^{2}}{\left(1-\mu^{2}\right)} \frac{a_{l} a_{s}}{a_{l}-a_{s}} h$,

where $E, \mu, U_{x x}$ are Young's modulus, Poisson's ratio and epitaxial layer strain, respectively; $a_{l}, a_{s}$ are the lattice parameters of the epitaxial layer and substrate, respectively, $h$ is the epitaxial layer thickness. The factor $a_{l} a_{s} /\left(a_{l}-a_{s}\right)$ is the misfit dislocation period [14]. The obtained critical thickness (Fig. 2) is $50 \mathrm{~nm}$ for growth on (100) InAs substrate and $54 \mathrm{~nm}$ in the case of (111) substrate orientation. These values are in a good agreement with the experimental results from [12] based on X-ray scattering analysis of misfit dislocation density.

Minimal supercooling related with the necessity to compensate the influence of elastic strains can be defined from the equality of chemical potentials of solid

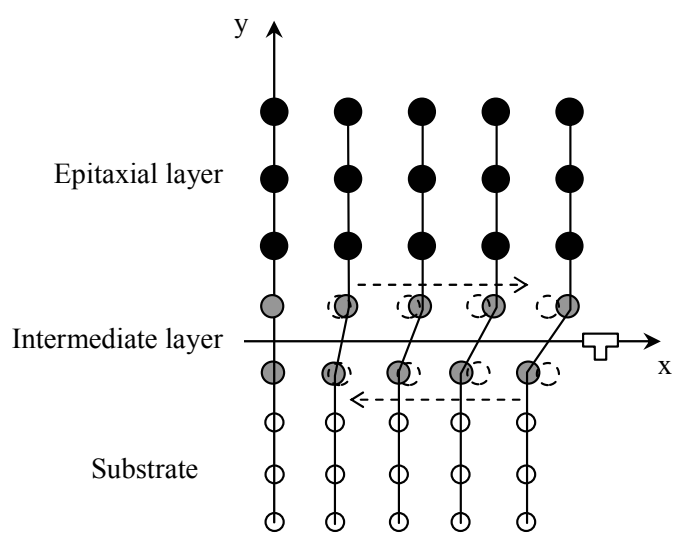

Fig. 1. Initial GaSb/InAs heterostructure for simulation. and liquid phases in the regular solution approach. When considering $\Delta T_{\min }<<T_{0}$, one can obtain

$$
\frac{1}{2} \frac{E U_{x x}^{2}}{(1-\mu)} \Omega=k_{\mathrm{B}} T_{0} \ln \left(\frac{\gamma\left(T_{0}\right) C\left(T_{0}\right)}{\gamma\left(T_{0}-\Delta T_{\min }\right) C\left(T_{0}-\Delta T_{\min }\right)}\right),
$$

where $\Omega$ is the atomic volume, $k_{\mathrm{B}}$ is the Boltzmann constant, $T_{0}$ is the temperature of solution-melt saturation, $C(T), \gamma(T)$ are the concentration and activity coefficients of $\mathrm{GaSb}$ in gallium melt at the temperature $T$. Based on dependence [15],

$$
R T \ln \gamma=\alpha_{\mathrm{GaSb}}\left(1-x_{\mathrm{GaSb}}\right)^{2},
$$

where $R$ is the absolute gas constant, $\alpha_{\mathrm{GaSb}}, x_{\mathrm{GaSb}}$ are the parameters of interphase interaction and mole fraction of $\mathrm{GaSb}$ in gallium melt and assuming $x_{\mathrm{GaSb}}<<1$, Eqs (2), (3) lead to the transcendent equation with respect to $\Delta T_{\min }$ :

$$
\begin{aligned}
& \frac{1}{2} \frac{E U_{x x}^{2}}{(1-\mu)} \Omega=k_{\mathrm{B}} T_{0}\left(\ln \frac{C\left(T_{0}\right)}{C\left(T_{0}-\Delta T_{\min }\right)}+\right. \\
& \left.+R^{-1}\left[\frac{\alpha_{\mathrm{GaSb}}\left(T_{0}\right)}{T_{0}}-\frac{\alpha_{\mathrm{GaSb}}\left(T_{0}-\Delta T_{\min }\right)}{T_{0}-\Delta T_{\min }}\right]\right),
\end{aligned}
$$

Using the approximation of antimony atomic fraction dependence in gallium melt [16] on the temperature close to the saturation one $T_{0}=450{ }^{\circ} \mathrm{C}, \quad x_{\mathrm{Sb}}=$ $=1.01 \cdot 10^{-9}+10^{0.0098 T}$ and the parameter of interphase interaction dependence $\alpha_{\mathrm{GaSb}}=(14738-21.48 T) \mathrm{J} / \mathrm{mol}$ [15], it can be found from (4) that the minimal supercooling temperatures are 7.8 and $5.8^{\circ} \mathrm{C}$ for $(111)$ and (100) orientations, respectively. These values are quite reachable during the epitaxy of $\mathrm{GaSb}$ layers, because the critical supercooling values of transition to labile region for antimony solution in gallium melts is about $12{ }^{\circ} \mathrm{C}$ [17].

Let us consider the influence of mechanical strains on growing epitaxial layer surface. In [8], the surface distortions induced by mechanical strains are considered with the harmonic approach. The highest rate of amplitude increasing is

$\omega_{m}=\frac{27 D n_{0} \Omega^{2} \sigma_{0}^{8} q_{m}^{3}}{2 E^{4} \alpha^{3} k_{\mathrm{B}} T}$,

where $\sigma_{0}$ is the epitaxial layer strain, $D$ is the adatom diffusion coefficient, $n_{0}$ is the density of surface defects, $\alpha$ is the surface energy. Estimation of $\omega_{m}$ value by (5) shows that $\omega_{m}=0.4 \mathrm{~s}^{-1}$ (it was assumed that $n_{0}=$ $1.46 \cdot 10^{10} \mathrm{~cm}^{-2}, \alpha=0.364 \mathrm{~J} / \mathrm{m}^{2}, D=2.8 \cdot 10^{-5} \mathrm{~cm}^{2} / \mathrm{s}, \sigma_{0}=$ $\left.8.7 \cdot 10^{8} \mathrm{~Pa}\right)$. As a characteristic time of process the value $1 / \omega_{m}$ can be used [8]. Hence, for suppression of dislocations, it is necessary that the time of achieving the critical thickness, which leads to relaxation of considerable part of elastic strains due to misfit 

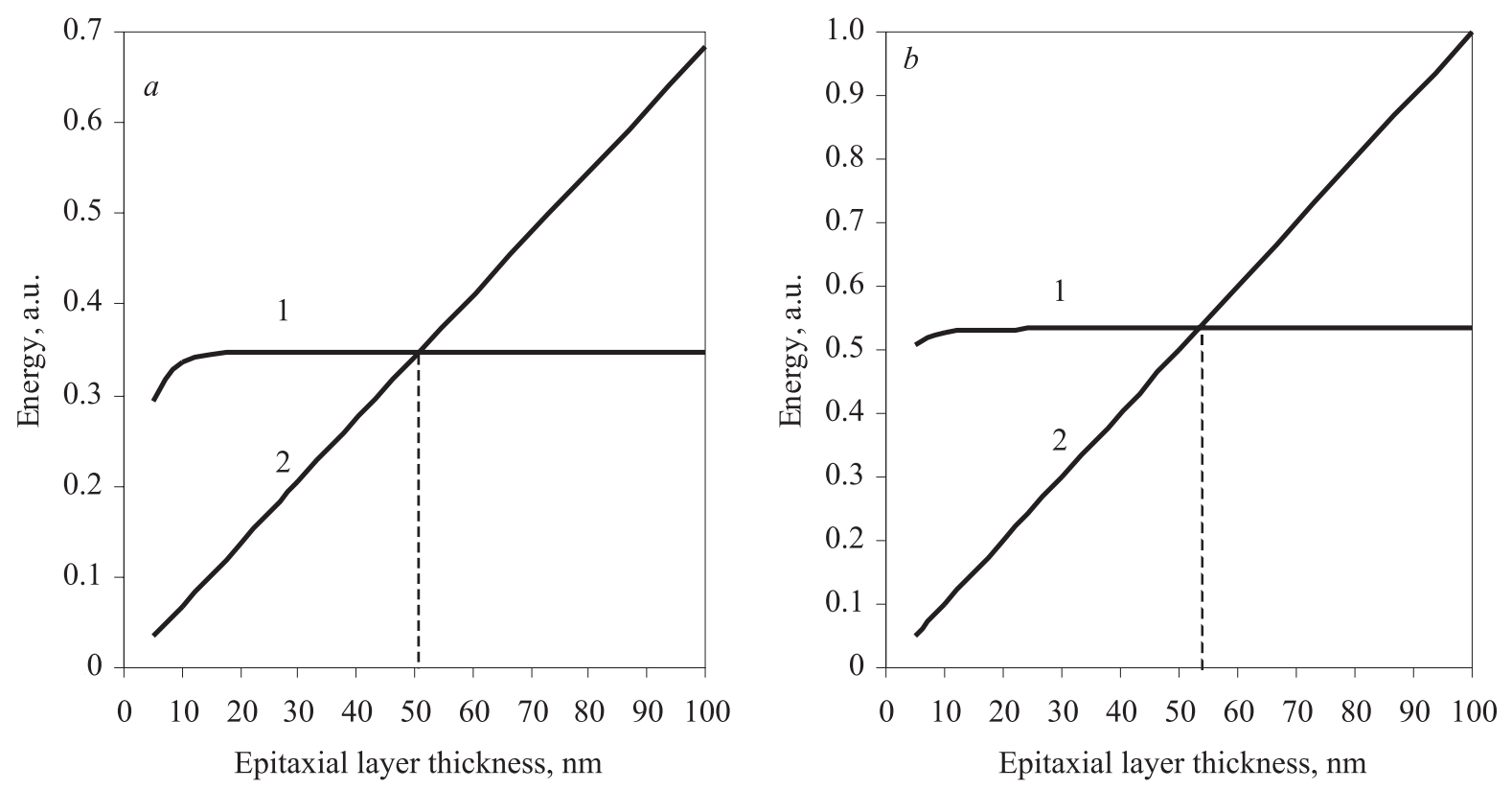

Fig. 2. Dependences of energy of structures that contains misfit dislocation (curves 1) and elastically strained dislocation-free epitaxial layer (curves 2) on the layer thickness, the layer being grown on the substrates with orientations (111) (a) and (100) (b).

dislocation formation, must be less than $1 / \omega_{m}=2.5 \mathrm{~s}$, hence, the growth rate must be higher than $22 \mathrm{~nm} / \mathrm{s}$.

\section{Experiment}

Calculated values of minimal supercooling and growth rate were used for GaSb heteroepitaxy on the (111) InAs substrate. The conditions for obtaining the planar layer were as follows:

$\left\{\begin{array}{l}7.8{ }^{\circ} \mathrm{C} \leq \Delta T<12{ }^{\circ} \mathrm{C}, \\ h(2.5 \mathrm{~s})>55 \mathrm{~nm} .\end{array}\right.$

Morphology of epitaxial layers obtained by (6) and at $\Delta T=5{ }^{\circ} \mathrm{C}$ are shown in Fig. $3 \mathrm{a}$ and $\mathrm{b}$, respectively. In Fig. $3 \mathrm{a}$ shown are the continuous heteroepitaxial layer obtained under fulfiment of the conditions (6). The layer roughness was less than $0.3 \mu \mathrm{m}$ (layer thickness was about $3 \mu \mathrm{m}$ ). The deviation from the conditions (6) led to pronounced island growth that resulted in the non-planar epitaxial layer surface (Fig. 3b).
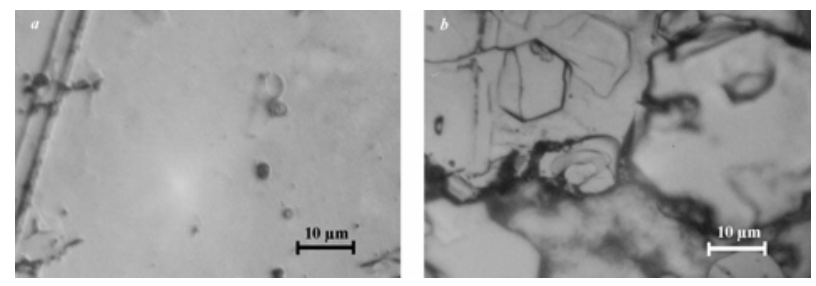

Fig. 3. Morphology of $\mathrm{GaSb} / \mathrm{InAs}$ (111) epitaxial layers obtained: a) adhering the conditions $(6)$; b) not adhering the latter (6).

\section{Conclusions}

Thus, influence of strains during the initial growth stages of $\mathrm{GaSb} / \mathrm{InAs}$ heterostructure on epitaxial layer planarity was considered. The availability of strains increases the chemical potential in the solid phase. To compensate this increase, it is necessary to provide the supercooling temperature higher than $7.8^{\circ} \mathrm{C}$ for $[111]$ and $5.8^{\circ} \mathrm{C}$ for [100] growth directions. Also, the elastic strains can cause distortion of epitaxial layer surface. To suppress all distortion modes the minimal growth rate must be $22 \mathrm{~nm} / \mathrm{s}$.

Such growth conditions must be provided until the epitaxial layer thickness reaches the critical thickness value for misfit dislocation formation when elastic strains considerably relax. The calculated critical thicknesses are $50 \mathrm{~nm}$ for [100] and $54 \mathrm{~nm}$ for [111] growth directions, respectively.

Experiment shows that adherence of calculated growth conditions causes obtaining the unbroken $\mathrm{GaSb}$ heteroepitaxial layer on the InAs substrate with a small roughness. Using the insufficient supercooling leads to an island growth mode.

\section{References}

1. M.J. Yang, C.H. Yang, B.R. Bennett, and B.V. Shanabrook, Evidence of a hybridization gap in "semimetallic" InAs/GaSb systems // Phys. Rev. Lett. 78, p. 4613-4616 (1997).

2. G.J. Conibeer, C.W. Bumby, R.J. Nicholas, and N.J. Mason, Towards a GaSb-InAs tandem junction TPV cell // Proc. of $17^{\text {th }}$ European PV Solar Energy Conf. and Exhibition, Munich, Germany, 2001, p. 175-179. 
3. B.Z. Nosho, B.R. Bennett, L.J. Whitman, M. Goldenberg, Effects of $\mathrm{As}_{2}$ versus $\mathrm{As}_{4}$ on InAs/GaSb heterostructures: As-for-Sb exchange and film stability // J. Vac. Sci. Technol. B. 19(4), p. $1626-1630$ (2001).

4. M.G. Mauk, V.M. Andreev, GaSb-related materials for TPV cells // Semicond. Sci. Technol. 18(5), p. S191-S201 (2003).

5. J.R. Skelton and J.R. Knight, Liquid-phase epitaxy of $\mathrm{In}(\mathrm{As}, \mathrm{Sb})$ on $\mathrm{GaSb}$ substrates using antimony-rich melts // Solid-State Electronics 28(11), p. 1166-1168 (1985).

6. I.Ye. Maronchuk, V.V. Kurak, E.V. Andronova, and Ye.A. Baganov, Obtaining GaSb/InAs heterostructures by liquid phase epitaxy // Semicond. Sci. Technol. 19, p. 747-751 (2004).

7. A.A. Chernov, Modern crystallography, Vol. III, Crystal Growth. Springer, Berlin (1984).

8. D.G. Cahill, Morphological instabilities in thin-film growth and etching // J. Vac. Sci. Technol. A. 21, p. S110-S116 (2003).

9. H. Gao, W.D. Nix. Surface roughening of heteroepitaxial thin films // Annu. Rev. Mater. Sci. 29, p. 173-209 (1999).

10. J.W. Matthews and A.E. Blakeslee, Defects in epitaxial multilayers. I. Misfit dislocations // J. Crystal Growth 27, p.118-125 (1974).
11. B.R. Bennett, Strain relaxation in $\mathrm{InAs} / \mathrm{GaSb}$ heterostructures // Appl. Phys. Lett. 73(25), p. 37363738 (1998).

12. A.Y. Babkevich, R.A. Cowley, N.J. Mayson, S. Sandiford and A. Stunault, X-ray scattering from epitaxial $\mathrm{GaSb} / \mathrm{InAs}$ thin films below and above the critical thickness // J. Phys.: Condens. Mat.14, p. 7101-7121 (2002).

13. M.G. Mil'vidskij, V.B. Osvenskij, Structural defects in semiconductor epitaxial layers. Metallurgija, Moscow (1985).

14. J.H. Van-der-Merwe, Crystal interfaces // J. Appl. Phys. 34, p. 117-127 (1963).

15. J.C. DeWinter, M.A. Pollack, A.K. Srivastava, J.L. Zyskind, Liquidus measurements of $\mathrm{Ga}-\mathrm{Sb}$ and In-As in the $375-650{ }^{\circ} \mathrm{C}$ range // J. Appl. Phys. 59(10), p. 3593-3595 (1986).

16. R. Hall, Solubility of III-V compound semiconductors in column III liquids, in: Technology of semiconductor compounds. Ed. A.Y. Nashel'skij. Metallurgija, Moscow (1967) (in Russian).

17. L.S. Lunin, M.L. Lunina, A.V. Blagin, O.E. Draka, Liquid phase crystallization of multiplayer $\mathrm{InSb}_{1-\mathrm{x}} \mathrm{Bi}_{\mathrm{x}} / \mathrm{InSb}$ and $\mathrm{GaSb}_{1-\mathrm{x}} \mathrm{Bi}_{\mathrm{x}} / \mathrm{GaSb}$ heterostructures. Proc. XI National Conference on Crystal Growth (Moscow, Russia). http://ns.crys.ras.ru/nccg/ REPORTS/lls1_1.html (in Russian). 